\title{
Aromatherapy massage: a promising non-pharmacological adjuvant treatment for osteoarthritis knee pain
}

Reza Ganji

Department of Orthopedic Surgery, School of Medicine, North Khorasan University of Medical Sciences, Bojnurd, Iran

\section{LETTER TO THE EDITOR}

Knee osteoarthritis $(\mathrm{OA})$ is a common chronic degenerative joint disease that commonly affects older adults. Pain is a major and disabling symptom for patients with knee OA. Therefore, relieving the knee pain and its functional improvement are the primary therapeutic goal in these patients [1].

Although a range of therapeutic modalities have been investigated for pain control in patients with knee OA, pain continues to remain a major unresolved challenge in these patients. Unfortunately, using pharmacological treatments such as nonsteroidal anti-inflammatory drugs (NSAIDs) and cyclooxygenase-2 (COX2) inhibitors, as a mainstay for pain control in knee OA, is not without adverse effects [2]. It is believed that using the combination of non-pharmacologic and pharmacologic approaches for pain management in patients with knee OA could help to prevent or mitigate adverse events induced by analgesic drugs, while providing therapeutic benefit $[3,4]$.

Recently, aromatherapy massage has been proposed as a promising alternative adjunctive therapeutic modality for pain control in patients with knee OA. Although the efficacy of aromatherapy for pain management has been relatively well known, aromatherapy massage for chronic pain management is relatively new and still developing [5].

In a study by Efe Arslan et al. [6] has been shown that aromatherapy massage, compared to conventional massage and usual care, significantly and efficiently reduce the knee pain and morning stiffness in patients with $\mathrm{OA}$ of the knee. Another study by Nasiri et al. [7] revealed the statistically significant effectiveness of lavender essential oil aromatherapy massage for pain control, immediately and 1 week after intervention, in patients with knee OA. However, this effectiveness was not statistically significant at 4 weeks after intervention. Due to the lack of long-term effectiveness of aromatherapy massage in this study, further long-term studies are needed to determine if the pain-relieving effects of this modality in patients with knee $\mathrm{OA}$ are long-lasting.

In a case study which has been conducted on $32 \mathrm{pa-}$ tients with bilateral grade two knee OA (presence of definite osteophytes and possible narrowing of the joint space on plain radiography of the knee) has been demonstrated that lavender oil aromatherapy massage, four days a week for one month, significantly reduced the patients' knee pain, tenderness, and morning stiffness [8]. A study by Won and Chae [9] revealed the significant effect of ar-

Received August 13, 2018. Revised September 4, 2018. Accepted September 7, 2018.

Correspondence to: Reza Ganji

Department of Orthopedic Surgery, School of Medicine, North Khorasan University of Medical Sciences, Bojnurd 94149-75519, Iran Tel: +98-58-3151, Fax: +98-58-3224-6144, E-mail: drrezaganji@yahoo.com ORCID: https://orcid.org/0000-0002-7198-3767

() This is an open-access article distributed under the terms of the Creative Commons Attribution Non-Commercial License (http:// creativecommons.org/licenses/by-nc/4.0/), which permits unrestricted non-commercial use, distribution, and reproduction in any medium, provided the original work is properly cited.

(C) The Korean Pain Society, 2019 
omatherapy massage, twice a week for four weeks, in reducing knee pain in elderly patients with $\mathrm{OA}$ of the knee. In terms of safety, none of the abovementioned studies have reported any serious adverse events related to application of aromatherapy massage in patients with knee $\mathrm{OA}$.

Due to the growing popularity of complementary and alternative medicine, as well as the potential benefit of aromatherapy massage for pain management in patients with knee OA, it seems that aromatherapy massage can be used as a safe, simple, and effective alternative adjunctive therapeutic modality for pain control in patients with knee OA. Further well-design studies are required to evaluate and determine the optimal type and concentration of essential oils, duration of the treatments, and number of sessions of aromatherapy massage for better pain control in patients with knee OA.

\section{CONFLICT OF INTEREST}

No potential conflict of interest relevant to this article was reported.

\section{REFERENCES}

1. Ganji R, Pakniat A, Armat MR, Tabatabaeichehr M, Mortazavi $H$. The effect of self-management educational program on pain intensity in elderly patients with knee osteoarthritis: a randomized clinical trial. Open Access Maced J Med Sci 2018; 6: 1062-6.

2. Alshami AM. Knee osteoarthritis related pain: a narrative review of diagnosis and treatment. Int J Health Sci (Qassim) 2014; 8: 85-104.

3. Rannou F, Poiraudeau S. Non-pharmacological approaches for the treatment of osteoarthritis. Best Pract Res Clin Rheumatol 2010; 24: 93-106.

4. Hasanzadeh Kiabi F, Habibi MR, Soleimani A, Emami Zeydi A. Mirror therapy as an alternative treatment for phantom limb pain: a short literature review. Korean J Pain 2013; 26: 309-11.

5. Lakhan SE, Sheafer $H$, Tepper D. The effectiveness of aromatherapy in reducing pain: a systematic review and meta-analysis. Pain Res Treat 2016; 2016: 8158693.

6. Efe Arslan D, Kutlutürkan S, Korkmaz M. The effect of aromatherapy massage on knee pain and functional status in participants with osteoarthritis. Pain Manag Nurs 2019; 20: 62-9.

7. Nasiri A, Mahmodi MA, Nobakht Z. Effect of aromatherapy massage with lavender essential oil on pain in patients with osteoarthritis of the knee: a randomized controlled clinical trial. Complement Ther Clin Pract 2016; 25: 75-80.

8. Zhang R, Kerrin TL, Zikri EN. Evaluation of the effect of aromatherapy in management of knee osteoarthritis patients. Int J Complement Altern Med 2018; 11: 78-80.

9. Won SJ, Chae YR. The effects of aromatherapy massage on pain, sleep, and stride length in the elderly with knee osteoarthritis. J Korean Biol Nurs Sci 2011; 13: 142-8. 